\title{
Swarm Robotics: Collective Behavior Inspired by Nature
}

Ying Tan*

\section{Department of Intelligence Science, School of Electronic Engineering and Computer Science, Peking University, Beijing, China}

\section{Definition of Swarm Robotics}

Swarm robotics is a new approach to the coordination of multirobot systems which consist of a number of simple physical robots [1]. It is supposed that a desired collective behavior emerges from the interaction between the robots and the interaction of robots with the environment. This approach emerged in the field of artificial swarm intelligence as well as the biological study of insects, ants and other fields in the nature, where a swarm behavior occurs. The aim of researches of swarm robotics is to study the design of large amount of relatively simple robots, their physical bodies and their controlling behaviors. The individuals in the swarm are normally simple, small and low cost so as to take the advantage of a large population. A key component of the system is the communication between the agents in the group which is generally local and no center, and guarantees the system is scalable and robust.

A plain set of rules at individual level can produce a large set of complex behaviors at the swarm level. The rules of controlling the individuals are abstracted from the cooperative behavior of a swarm in nature. As the swarm is distributed and de-centralized, so the system shows high efficiency, parallelism, scalability and robustness.

\section{Characteristics of Swarm Robotics}

Since the swarm robotics is mostly inspired from the nature swarms, it's a good reference for analyzing the characteristics of swarm robotics through the characteristics of nature swarms. Counting from the first attempt on a swarm of intelligent agents, the research of swarm robotics started a century ago, taking the advantages of the nature swarm researches.

The first hypothesis of nature swarms is quite personified and assumes that each individual has a unique ID for cooperation and communication. The information exchange in the swarm is regarded as a centralized network. The queens in ant and bee colonies are supposed to be responsible for transmitting and assigning the information to each agent. However, S. Jha et al. [2] proved that the network in the swarm is decentralized. Thanks to the researches in recent half century, the biologists can now assert that there are no unique ID or other globally storage information in the network. No single agent can access to all the information in the network and a pacemaker is therefore inexistent.

The biologists now believe that the social swarms are organized as a decentralized system distributed in the whole environment which can be described through a probabilistic model. The agents in the swarm follow their own rules according to local information. The behaviors of the group emerge from these local rules which affect the information exchange and topology structure in the swarm. The rules are also the key component to keep the whole structure to be flexible and robust even when the sophisticated behaviors are emerged.

\section{Application Scopes of Swarm Robotics}

The potential applications of swarm robotics include the tasks that demand the miniaturization, like distributed sensing tasks in micro machinery or the human body. On the other hand, the swarm robotics can be suited to the tasks that demand the cheap designs, such as mining task or agricultural foraging task. The swarm robotics can be also involved in the tasks that require large space and time cost, and are dangerous to the human being or the robots themselves, such as postdisaster relief, target searching, military applications, etc.

The study of robotics application in target search has grown substantially in the recent years. It is more preferable for the dangerous or inaccessible working area. The problems involved in swarm robotics research can be classified into two classes. One class of the problems is mainly based on the patterns, such as aggregation, cartography, migration, self-organizing grids, deployment of distributed agents and area coverage. Another class of problems focuses on the entities in the environment, e.g. searching for the targets, detecting the odor sources, locating the ore veins in wild field, foraging, rescuing the victims in disaster areas and etc. Besides these problems, the swarm robotics can also be involved into more sophisticated problems, mostly hybrid of these two classes, including cooperative transportation, demining, exploring a planet and navigating in large area.

Several potential application scopes for swarm robotics systems which are very suitable are described below, classified as mainly four types.

\section{Tasks cover large area}

Swarm robotics system is distributed and specialized for the tasks requiring a large area of space, e.g. the tasks cover large areas. An easy example would be searching and collecting multiple targets in an open area [3]. The swarm tries to search with group cooperation to accelerate the search. The area may be very large and the swarm can take advantage of the parallel searching with several small groups within the sensing ranges of the robots.

In another scenario, the robots in the swarm are distributed in the environment and can detect the dynamic change of the entire area, such as chemical leaks or pollution. The swarm robotics can complete such tasks in a better way than sensor network since each robot can patrol in an area rather than stay still. This means that the swarm can monitor the area with fewer individuals. Besides monitoring, the robots in the swarm can locate the source, move towards the area and take quick actions. In an urgent case, the robots can aggregate into a patch to block the source as a temporary solution. Another example.

\section{Tasks dangerous to robot}

Thanks to the scalability and stability, the swarm provides

*Corresponding author: Ying Tan, Department of Intelligence Science, School of Electronic Engineering and Computer Science, Peking University, Beijing 100837, China, Mobile: 86-13581667196; Fax: 86-10-62767611; E-mail: ytan@pku.edu.cn

Received October 21, 2013; Accepted October 22, 2013; Published October 26 2013

Citation: Tan Y (2013) Swarm Robotics: Collective Behavior Inspired by Nature. J Comput Sci Syst Biol 6: e106. doi:10.4172/jcsb.1000e106

Copyright: (c) 2013 Tan Y. This is an open-access article distributed under the terms of the Creative Commons Attribution License,which permits unrestricted use, distribution, and reproduction in any medium, provided the original author and source are credited. 
redundancy for dealing with dangerous tasks. The swarm can suffer loss of robots to a great extent before the job has to be terminated. The robots are very cheap and are preferred for the areas which probably damage the workers. In some tasks, the robots may be irretrievable after the task, and the use of complex and expensive robots are thus economically unacceptable while the swarm robotics with cheap individuals can provide the reasonable solutions. For example, Murphy et al. [4] summarized the usage of robotics in mine rescue and recovery. They pointed out that although several applications already in use, the robots are beyond the requirement to show a desired performance in the tough environment under the ground. They proposed 33 requirements for the robots so as to achieve an acceptable behavior.

\section{Tasks require scaling population}

Workload of some tasks may change over time, and the swarm size should be scaled based upon the current workload for high efficiency in both time and economics. For example, in the task of clearing oil leakage after tank accidents, the swarm should maintain a high population when the oil leaks fast at the beginning of the task and gradually reduce the robots when the leak source is plugged and the leaking area is almost cleared. The swarm also scales among different regions if the progress of these regions becomes unbalanced.

Stormont [5] described the potential for using the swarms of autonomous robots to react a disaster site in the first 24 hours. He summarized the swarm that have the highest probability of finding survivors, and made some suggestions for future research in this area.

\section{Tasks require redundancy}

Robustness in the swarm robotics systems mainly benefits from the redundancy of the swarm, i.e. removing some robots does not have a significant impact on the performance. Some tasks focus on the result rather than the process, i.e. the system should make sure that the task will be completed successfully, mostly in the way of increasing redundancy.

\section{Swarm Robotics Systems in Real Life}

In the recent years, the researchers from especially American and European institutes have already utilized the swarm robotics in several real-life applications including most of the tasks mentioned above. Besides, researchers also proposed several simulation programs on computer to aid their researches, such as Player/Stage, Swarmanoid Simulation and etc.

MIT's Senseable City Lab developed a fleet of low-cost oil absorbing robots called Seaswarm [6] for ocean-skimming and oil removal. A nanomaterial robot can absorb oil up to 20 times of its weight. The system provides an autonomous solution for ocean environment protection.

Roombots [7] is a novel self-reconfiguring modular robotic system. The autonomous modular robots can assembly into robot that can alter its shape to adapt to a given task and working environment, such as self-assembly and reconfiguration of static objects like furniture in the day-to-day environment.
Formica [8] is a scalable, biologically-inspired swarm robotics platform. Its novel mechanical design permits production on standard circuit board assembly lines. The system takes the advantage of small cheap, long-life robots, supporting the peripherals, and can be scaled to a population with several hundred individuals. Scientists believe such swarms are suitable solutions for the tasks like Mars reconnaissance, earthquake recovery, etc.

Swarm robotics can be useful for military application as well. Pettinaro et al. [9] proposed a self-reconfigurable robot system for foraging, searching and rescuing, which has the ability to cope with occasionally failure. Military experts believe that the bionic aero vehicles inspired from swarm intelligence technology will become applicable in a few years. It can be foreseen that machine bees or cockroaches with reconnaissance equipment and bombs will possibly show up in future war.

(This work is supported by NSFC with Grant No. 61375119).

\section{References}

1. Zheng $Z$, Tan $Y$ (2013) Research advance in swarm robotics. Defence Technology 9: 33-59.

2. Shalene Jha, Rowan GCF, Jes SP, Thomas GP, Rita C, et al. (2006) The queen is not a pacemaker in the small-colony wasps polistes instabilis and $p$. dominulus. Animal behaviour 71: 1197-1203

3. Zheng Z, Tan $Y$ (2013) Group explosion strategy for searching multiple targets using swarm robotic. In IEEE Congress on Evolutionary Computation, 2013. 821-828.

4. Robin RM, Kravitz J, Samuel LS, Shoureshi R (2009) Mobile robots in mine rescue and recovery. Robotics \& Automation Magazine, IEEE.16: 91-103.

5. Daniel P Stormont (2005) Autonomous rescue robot swarms for first responders Proceedings of the 2005 IEEE International Conference on Computational Intelligence for Homeland Security and Personal Safety, IEEE. 151-157.

6. Homepage of seaswarm project.

7. Alexander Spröwitz (2010) Roombots: design and implementation of a modular robot for reconfiguration and locomotion. Lausanne: École Poly technique Fédérale de Lausanne, 2010.

8. Stephen E, Jeffrey G, Alexis J, Robert S, Joanna AS (2008) A swarm robotics project. Technical report, University of Southampton.

9. Giovanni CP, Ivo WK, Luca MG, Francesco, Floreano D, et al. (2002) Swarm robotics: a different approach to service robotics. In Proceedings of the 33rd International Symposium on Robotics. 71-76. 